\title{
Experimental Study on Temperature Field Evolution Mechanism of Artificially Frozen Gravel Formation under Groundwater Seepage Flow
}

\author{
Tian-liang Wang $\mathbb{D}^{1,2,3}$ Fei Zhang $\mathbb{D}^{2,3}$ Yang Wang $\mathbb{D}^{2,3}$ Zhen $W u\left(\mathbb{D},{ }^{5}\right.$ Ya-meng $H e \mathbb{D}^{4}$, \\ and Zu-run Yue ${ }^{1}{ }^{1}$ \\ ${ }^{1}$ State Key Laboratory of Mechanical Behaviour and System Safety of Traffic Engineering Structures, \\ Shijiazhuang Tiedao University, Shijiazhuang 050043, China \\ ${ }^{2}$ Key Laboratory of Roads and Railway Engineering Safety Control of Ministry of Education, Shijiazhuang Tiedao University, \\ Shijiazhuang 050043, China \\ ${ }^{3}$ School of Civil Engineering, Shijiazhuang Tiedao University, Shijiazhuang 050043, China \\ ${ }^{4}$ State Key Laboratory for Geomechanics \&Deep Underground Engineering, China University of Mining and Technology, \\ Xuzhou 221116, China \\ ${ }^{5}$ Jinan Design Institute, China Railway Engineering Consulting Group Co. Ltd., Jinan 250022, China
}

Correspondence should be addressed to Tian-liang Wang; wangtl@stdu.edu.cn and Zu-run Yue; yuezr@qq.com

Received 28 August 2021; Accepted 6 January 2022; Published 20 January 2022

Academic Editor: Marco Cannas

Copyright (c) 2022 Tian-liang Wang et al. This is an open access article distributed under the Creative Commons Attribution License, which permits unrestricted use, distribution, and reproduction in any medium, provided the original work is properly cited.

\begin{abstract}
Artificially ground freezing method is increasingly applied in formations with high permeability. The groundwater seepage flow should be considered because an excessive groundwater seepage flow would make the merging of the frozen wall challenging. Therefore, in this study, we investigate the temperature field and frozen wall merging characteristics at varying groundwater seepage flow rates in gravel formation. Results show that the heat exchange between the seepage flow and freezing pipes delays the merging of the frozen wall and reduces its total thickness. The groundwater seepage flow restricts the freezing of the upstream zone and accelerates the freezing of the downstream zone. The upstream and downstream temperature fields are symmetrical in nonseepage flow conditions but are asymmetrical in the presence of seepage flow. The merged frozen wall presents an arched shape and shifts to the downstream zone. The "scouring effect" and "water barrier effect" simultaneously act on the merging process of the frozen wall. The total thickness of the frozen wall decreases by more than $30 \%$ when the flow rate increases from 0 to $5.0 \mathrm{~m} / \mathrm{d}$. Optimising the layout of the freezing pipes in gravel formations is a reasonable solution for a safe and economical design.
\end{abstract}

\section{Introduction}

The artificially ground freezing (AGF) method originates from mining construction and has many advantages, including an adequate water blocking, high-soil strength, easy construction, and high-formation adaptability $[1,2]$. Further development of this method has led to its extensive employment both as a structural support system and as a water barrier in large-scale open excavations such as subway tunnels, foundation pits, and shaft constructions [3-5].
Engineering practices have also contributed to the improvement of the AGF theory, level of application, construction method, and other aspects of the AGF technique [6].

The AGF method applied in subway tunnels is initially used to enhance the strength of the soil, such as the excavation in saturated silty clay, mucky, and mucky clay with high-water content and compressibility [5, 7]. Currently, increasingly diverse ground formations are being prepared using the AGF method. It has been applied to sand, gravel, and pebble soil formation with high permeability and groundwater seepage 
flow. In contrast to the problem of frost heave and subsequent thaw-induced settlement in fine grained soil, the coupling problem of groundwater seepage flow and heat flow is more concerned in coarse-grained soil [8]. Engineering practices have shown that the groundwater seepage flow must be considered in the formations with high permeability because neglecting the groundwater seepage flow when planning the AGF process often leads to serious challenges in frozen wall merging and even disrupts the working schedule [4, 9-11].

The problem of AGF method applied in coarse-grained soils has attracted increased attention. Scholars have studied the effect of groundwater seepage flow on frozen walls by conducting physical modelling tests, numerical simulations, and theoretical analyses $[8,10,12,13]$. With the assistance of a large-scale physical model, Pimentel et al. [8] investigated the effect of seepage flow velocity on the frozen wall merging and thickness in a medium-coarse sand formation and provided an analytical solution to the frozen wall merging. Based on a hydrothermal modelling, Vitel et al. [12] presented the use of the thermohydraulic model and the freeze-pipe ground model to the Cigar Lake underground mine. Moreover, Marwan et al. [13] provided an optimal method to shorten the closure time of frozen wall in a sand formation subject to the effect of groundwater seepage flow. However, previous studies have paid more attention to fine sand and medium-coarse sand formations, which represent a relatively uniform formation. Research studies on gravel formations subject to the effect of seepage flow, including the characteristics of nonuniformity, large particles, and complex pore distribution of gravel soil, are currently lacking [14, 15].

Thus, to identify/quantify the specific temperature response of the gravel formation during the AGF process, several physical modelling experiments were conducted to visualize, discuss, and derive the coupling mechanism of the groundwater seepage flow and temperature field. In addition, the effects of the groundwater seepage flow rate on the merge of the frozen wall are analysed. Finally, issues regarding the frozen wall merging and layout optimisation of the freezing pipes are discussed.

\section{Design of Physical Modelling Experiment}

2.1. Similarity Law and Selection of Similarity Constant. During the AGF process of gravel formations, the heat dissipated from the freezing pipes diffuses and affects the temperature field, thereby reducing the permeability of the surrounding gravel soil and affecting the groundwater seepage. The groundwater seepage flow would take away the heat of the freezing pipes and affect the merging of the frozen wall. Thus, the merging of the frozen wall is the result of the coupling effect between the groundwater seepage flow and gravel formation temperature field.

According to similarity theory, the physical quantities in coupling equations can be analysed using the similarity law, and the main parameters are determined as follows.

2.1.1. Geometric Similarity Constant. In the physical modelling experiment, a $25 \mathrm{~mm} \times 2.5 \mathrm{~mm}$ seamless steel pipe has been selected to simulate a $127 \mathrm{~mm} \times 8 \mathrm{~mm}$ freezing pipe extensively used in practical engineering, and the geometric similarity constant is set to 5 in consideration of the processability of the steel pipe and the size of the model. According to the similarity criterion $\pi_{1}$, the similarity constant of the freezing pipe spacing is 5 , and the freezing pipe spacing in the physical modelling experiment is set as $20 \mathrm{~cm}$ to simulate a prototype with a freezing pipe spacing of $100 \mathrm{~cm}$.

2.1.2. Material Similarity Constant. The tested soil investigated in this study is sampled from the Jinan Metro connected aisle. In the soil, the grain size of $80 \%$ of the particles is larger than $2 \mathrm{~mm}$, and the content of fines with a particle size smaller than $0.075 \mathrm{~mm}$ is approximately zero, as shown in Figure 1. In addition, the physical and thermal properties are tested and listed in Table 1. Therefore, the CGS is classified as poorly graded gravel soil, according to the Standard for the Engineering Classification of Soil (GB/T 50145-2007; Ministry of Construction of the People's [16]. As its basic physical properties remain unchanged, the material similarity constant is equal to one.

2.1.3. Temperature Similarity Constant. According to the Kosovich criterion, the temperature parameters in the physical modelling are in good agreement with that in the prototype, that is, 1 .

2.1.4. Freezing Duration Similarity Constant. Based on the similarity criterion, the freezing duration for one day in the physical modelling is equivalent to 25 days in the prototype.

2.1.5. Seepage Flow Rate Similarity Constant. Based on the similarity criterion, a groundwater seepage flow rate of $5 \mathrm{~m} / \mathrm{d}$ in the physical modelling is equivalent to $1 \mathrm{~m} / \mathrm{d}$ in the prototype.

2.2. Physical Modelling Experimental Apparatus. As shown in Figure 2, the physical modelling experiment apparatus, that is, self-developed, is composed of four systems: a physical modelling box, groundwater seepage system, freezing and temperature controlling system, and temperature monitoring system.

2.2.1. Physical Modelling Box. Given the influence range and boundary effects of the temperature and groundwater seepage flow, the length, width, and height of the physical modelling box are set to $1.2,0.8$, and $1.0 \mathrm{~m}$, respectively. In addition, the physical modelling box is composed of a water feeding zone, gravel soil filling zone, and water draining zone. Pebbles with a size of $10 \mathrm{~mm}$ are filled in the water feeding and water draining zones, and a perforated aluminium plate is placed between the gravel soil and water feeding zone to buffer the scour of the inlet groundwater flow and obtain more uniform seepage conditions. In addition, the groundwater flows from the bottom to the top. During the entire AGF process, thermal insulation cotton is wrapped around the box to isolate the influence of ambient temperature. 


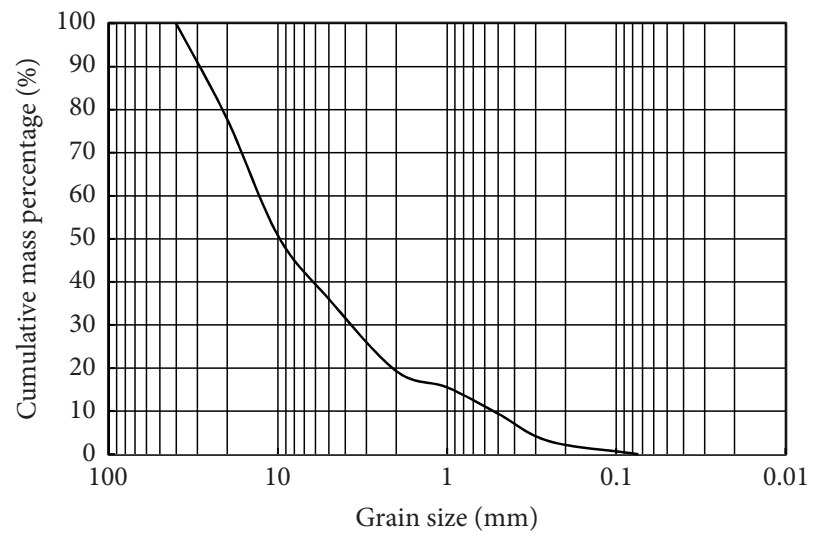

Figure 1: Particle size distribution of gravel soil.

TABLe 1: Physical and thermal properties of gravel soil.

\begin{tabular}{lcccccc}
\hline $\begin{array}{l}\text { Dry density } \\
\left(\mathrm{g} / \mathrm{cm}^{3}\right)\end{array}$ & Porosity & $\begin{array}{c}\text { Specific } \\
\text { gravity }\end{array}$ & $\begin{array}{c}\text { Permeability coefficient } \\
(\mathrm{m} / \mathrm{d})\end{array}$ & $\begin{array}{c}\text { Freezing } \\
\text { temperature }\left({ }^{\circ} \mathrm{C}\right)\end{array}$ & $\begin{array}{c}\text { Thermal conductivity } \\
\left(\mathrm{W} /\left(\mathrm{m}^{2} \cdot{ }^{\circ} \mathrm{C}\right)\right)\end{array}$ & $\begin{array}{c}\text { Specific volume heat } \\
\left(\mathrm{m} /\left(\mathrm{s} \cdot{ }^{\circ} \mathrm{C}\right)\right)\end{array}$ \\
\hline 1.883 & 0.3 & 2.69 & 60 & -0.04 & 1.07 & 1.30 \\
\hline
\end{tabular}

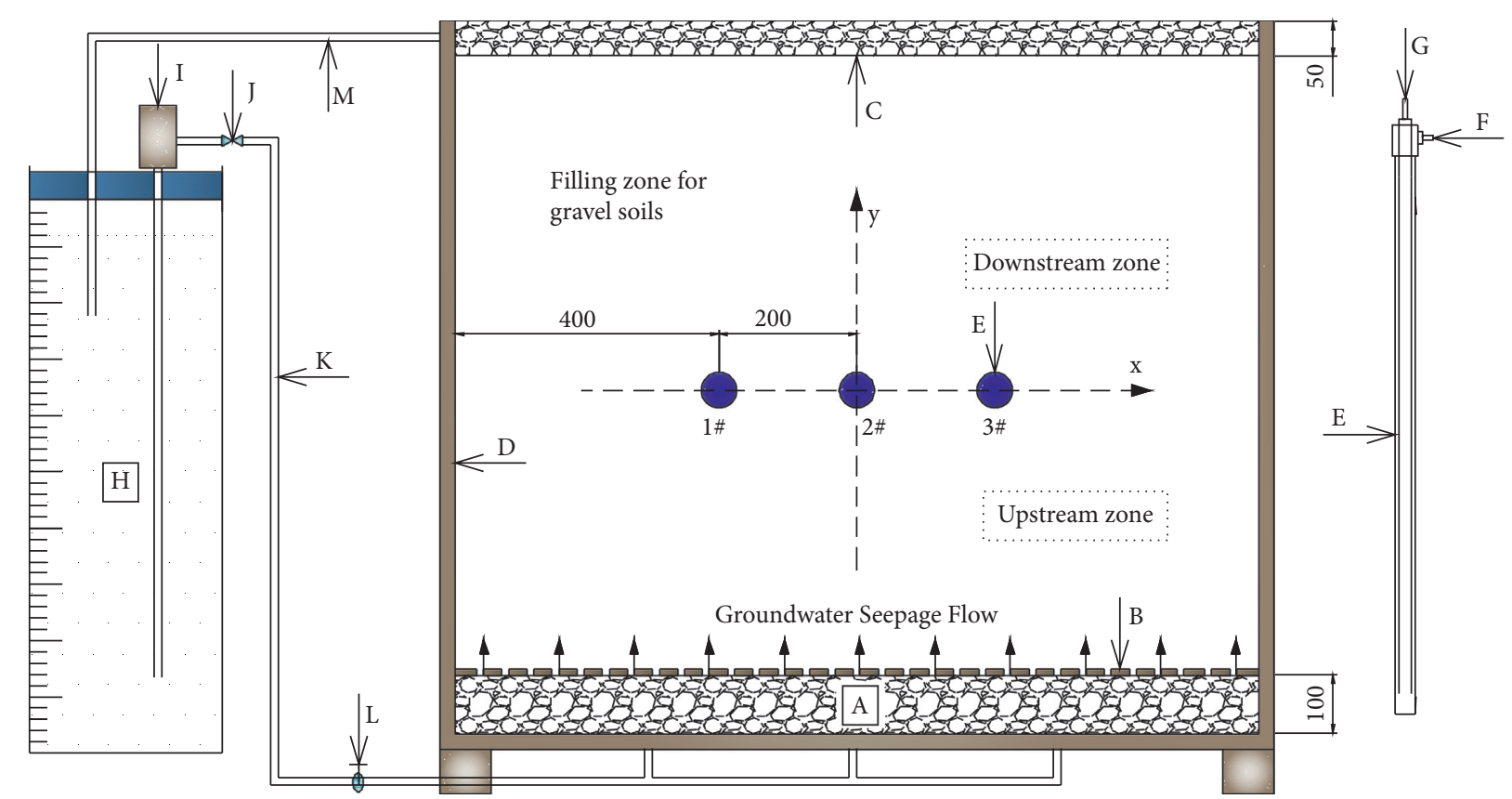

Figure 2: Schematic of the physical modelling experiment apparatus (unit: $\mathrm{mm}$ ): (A) water feeding zone filled with pebbles, (B) perforated aluminium plate, $(C)$ water draining zone filled with pebbles, (D) wall of physical modelling box, (E) freezing pipes, (F) brine outlet, $(\mathrm{G})$ brine inlet, $(\mathrm{H})$ thermostatic water tank, (I) power water pump, (J) water flow gauge, $(\mathrm{K})$ water feeding tube, (L) water flow valve, and $(\mathrm{M})$ water draining tube.

2.2.2. Groundwater Seepage System. The groundwater seepage system is composed of a thermostatic water tank connected with a NESLAB cold bath, power water pump, water flow gauge, water flow valve, water feeding tube, and water draining tube. The power water pump transports thermostatic water through the water feeding tube to the water feeding zone. The groundwater seepage flow rate is controlled by adjusting the water flow gauge and valve, and is defined as the flow at the water-crossing section in unit time.

2.2.3. Freezing and Temperature Controlling System. The freezing and temperature controlling system is composed of a NESLAB cold bath, a brine circulation tube, and freezing 
pipes, as shown in Figures 2 and 3. The freezing pipes are equipped with inner and outer casings to obtain a uniform temperature along the pipe wall. In addition, thermally insulation cotton is wrapped around the brine circulation tube to reduce the heat loss.

2.2.4. Temperature Monitoring System. Platinum resistance temperature sensors with an accuracy of $\pm 0.1^{\circ} \mathrm{C}$ and a data logging instrument are used for the real-time monitoring of the formation temperature. As shown in Figure 4, the physical modelling experiment is centred on the freezing pipes. Considering the location of the freezing pipes and the direction of the groundwater seepage flow, four temperature monitoring lines are arranged accordingly. Taking the L1 survey line (the $x$-axis) as the dividing line and considering the seepage flow direction of the groundwater, we can divide the entire crossing section into two zones: an upstream and a downstream zone.

2.3. Physical Modelling Experimental Scheme. Five groundwater seepage flow rates were investigated to study the merging process of the frozen wall in gravel formation. According to Section 2.1, Table 2 lists the experimental parameters in prototype and physical modelling.

Given these preliminary testing results, three freezing pipes are horizontally installed in the physical modelling box, and gravel soil is mixed and compacted layer-by-layer based on the dry density, as listed in Table 1. The temperature sensors are installed during the process, as shown in Figure 4. Once the gravel formation is prepared, the experimental apparatus is accurately assembled and the outside of the physical modelling box is wrapped with insulation cotton, as shown in Figure 2. In addition, the entire physical modelling experiment apparatus is placed in a high-low temperature chamber (length: $5.50 \mathrm{~m}$; width: $5.00 \mathrm{~m}$; height: $3.50 \mathrm{~m})$ to provide the constant ambient temperature of $20^{\circ} \mathrm{C}$.

Before the AGF process begins, the groundwater seepage flow and the formation temperature in the gravel formation are initialised to a constant state by adjusting the groundwater seepage system for $4 \mathrm{~h}$, as listed in Table 2 . After the constant state of groundwater seepage and temperature field is reached in the gravel formation, the AGF process is started by adjusting the freezing and temperature controlling system for $48 \mathrm{~h}$. Meanwhile, the formation temperature was automatically recorded by the data logger at intervals of $10 \mathrm{~min}$.

\section{Experimental Results and Analysis}

3.1. Temperature Evolution of the Brine and Freezing Pipes. When the groundwater seepage flows vertically through the freezing pipes, it will take away the heat of the brine and freezing pipes, and will lead to a heat loss, as shown in Figure 5. The entire process occurs in three stages: (1) the temperature decreases rapidly from 0 to $8 \mathrm{~h},(2)$ a supercooling stage is induced because of the latent heat of phase change (as illustrated in Wang et al. [17]), and (3) the temperature gradually decreases owing to the heat exchange and then levels off.

Subject to the premise of a constant working temperature of $-20^{\circ} \mathrm{C}$ and a cooling power of $850 \mathrm{~W}$, the final temperature of the brine increases with an increase in the groundwater seepage flow rate, and so does that of the freezing pipe. This indicates that the flowing water takes away the heat of the brine and freezing pipe, thus causing the temperature to rise. Moreover, the temperature difference between the brine and freezing pipe increases gradually. This indicates that the brine exchanges heat with the gravel soil and the water flowing through the freezing pipe. Therefore, the brine cannot reach the set temperature.

3.2. Temperature Distribution along the Seepage Flow Direction. Figure 6 shows the upstream and downstream temperature distributions of the gravel formation parallel to the seepage flow direction. As shown in Figure 4, the L3 survey line is located at the centre of the freezing pipes $1 \#$ and $2 \#$, and the L4 survey line is located at the freezing pipe 2\#. As illustrated, the gravel formation temperature is distributed symmetrically on both sides of the upstream and downstream in the case of nonseepage flow, and the heat provided by the freezing pipe is uniformly transmitted around the freezing pipe as the centre. However, the upstream temperature is evidently higher than the downstream temperature under the effect of the groundwater seepage flow. This is because the groundwater with temperature $20^{\circ} \mathrm{C}$ flows first through the upstream zone and then through the freezing pipes, carrying heat from the freezing pipe to the downstream zone. This type of asymmetry characteristic becomes more pronounced with an increase in the groundwater seepage flow rate. In addition, the closer we move to the freezing pipe, the lower the temperature will be.

In the upstream zone, the temperature at the same position increases with an incremental increase in the groundwater seepage flow rate, a particularly significant increase over the nonseepage case. This indicates that the groundwater seepage flow weakens the heat transfer of the freezing pipe to gravel formation in the upstream zone.

By contrast, the temperature at the same position decreases with an increase in the groundwater seepage flow rate and is approximately lower than the temperature of the nonseepage flow. This shows that the groundwater seepage flow carries more heat from the freezing pipe to the downstream zone that accelerates the freezing of the downstream zone around the freezing pipe.

3.3. Closure Time of Frozen Wall. To obtain the closure time of the frozen wall, the time history of the temperature at the survey points T1-6 is investigated, as shown in Figure 7. This survey point is located at the centre of the freezing pipes $1 \#$ and 2\# on the L1 survey line. As shown, the entire process is similar to that of the freezing pipe, as shown in Figure 5(b). The temperature increases as the groundwater seepage flow rate increases for the same freezing duration.

When the temperature at the survey points T1-6 reaches $-0.04^{\circ} \mathrm{C}$, it is taken as the approximate time for the merging of 


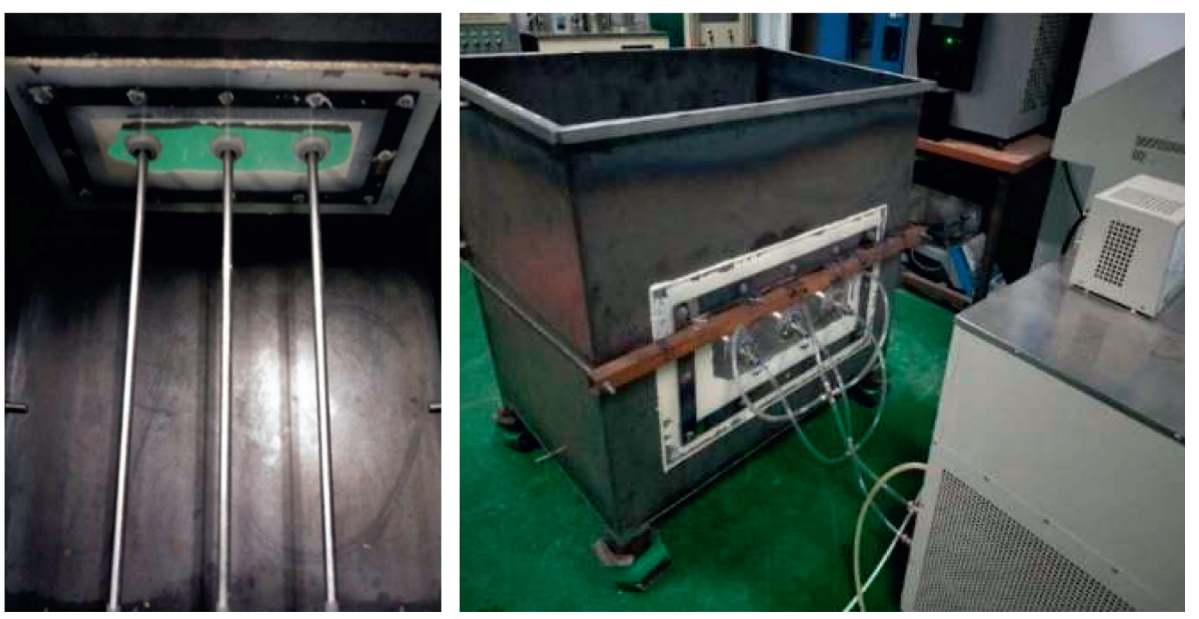

FIgURE 3: Freezing pipes (a) and physical modelling box (b).

TABLE 2: Experimental parameters in prototype and physical modelling.

\begin{tabular}{|c|c|c|c|c|}
\hline $\begin{array}{l}\text { Initial formation } \\
\text { temperature }\left({ }^{\circ} \mathrm{C}\right)\end{array}$ & Arrangement of freezing pipes & $\begin{array}{l}\text { Temperature of the brine in } \\
\text { freezing pipes }\left({ }^{\circ} \mathrm{C}\right)\end{array}$ & $\begin{array}{l}\text { Groundwater seepage } \\
\text { flow rate }(\mathrm{m} / \mathrm{d})\end{array}$ & $\begin{array}{c}\text { Freezing } \\
\text { duration }(\mathrm{h})\end{array}$ \\
\hline $20 /(20)$ & $\begin{array}{l}\text { Three freezing pipes are arranged } \\
\text { horizontally at intervals of } 20 \mathrm{~cm} /(100 \mathrm{~cm})\end{array}$ & $-20 /(-20)$ & $\begin{array}{c}0 \text { (saturated) } \\
1.25 /(0.25) \\
2.50 /(0.50) \\
3.75 /(0.75) \\
5.00 /(1.00)\end{array}$ & $48 /(1200)$ \\
\hline
\end{tabular}

Note. The parameters listed in the bracket ( ) are the prototype parameters in this study.

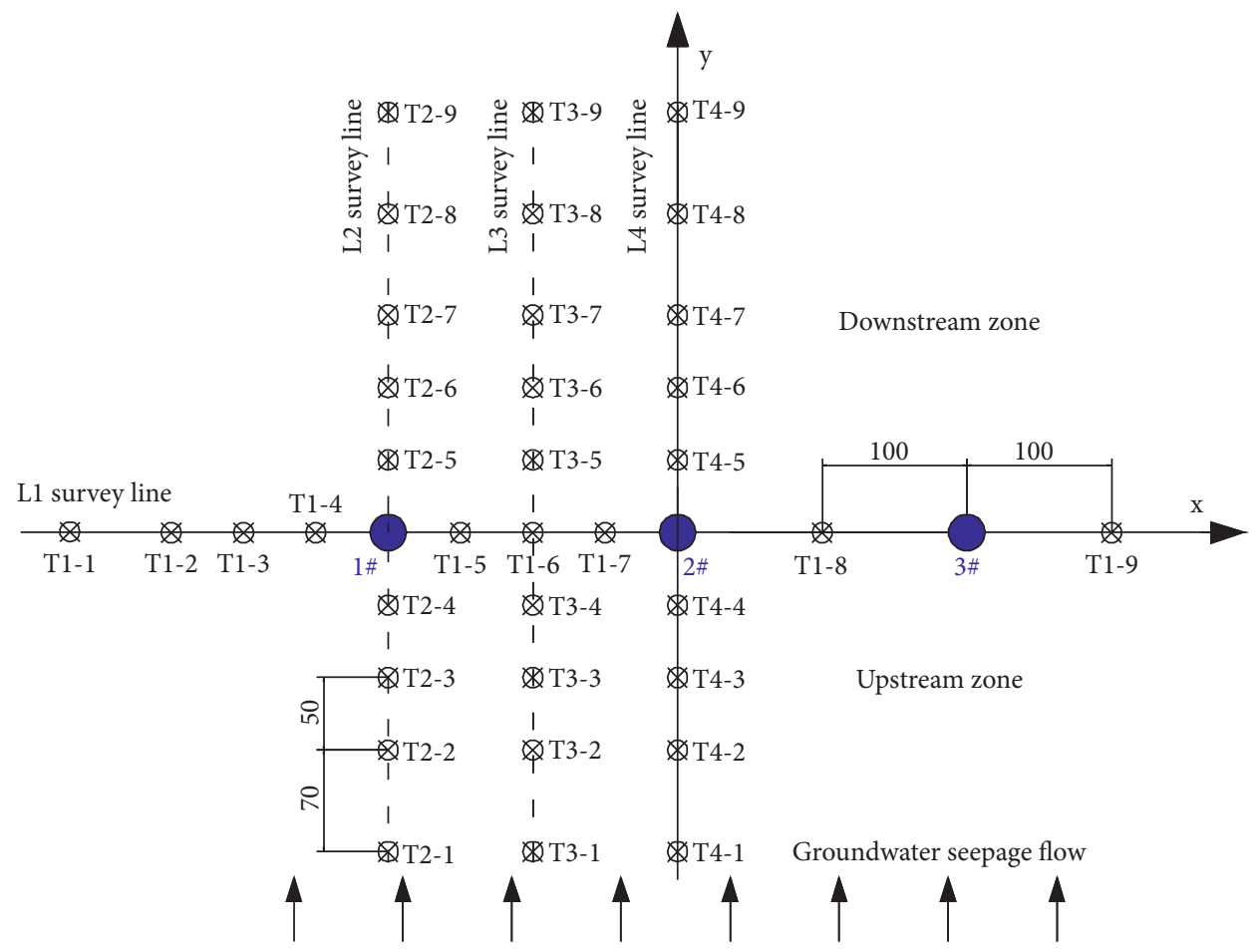

Figure 4: Arrangement of temperature sensors and freezing pipes. 


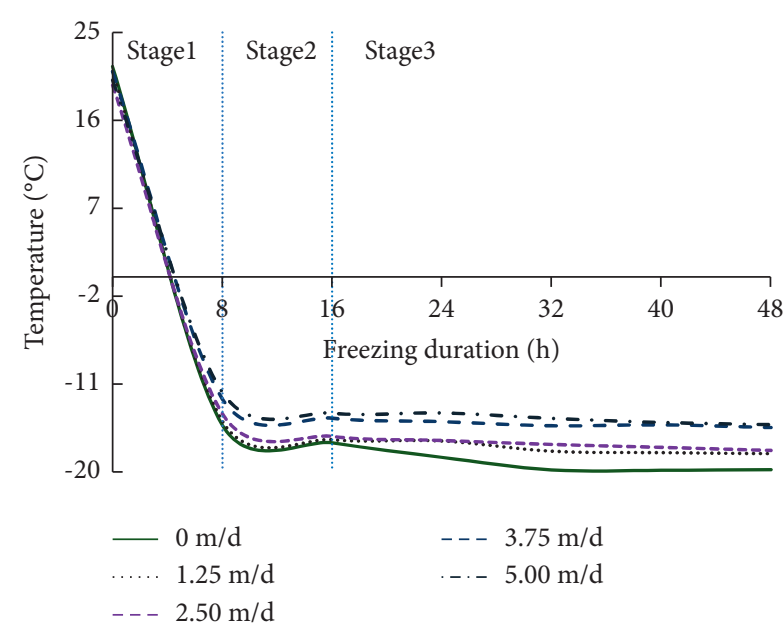

(a)

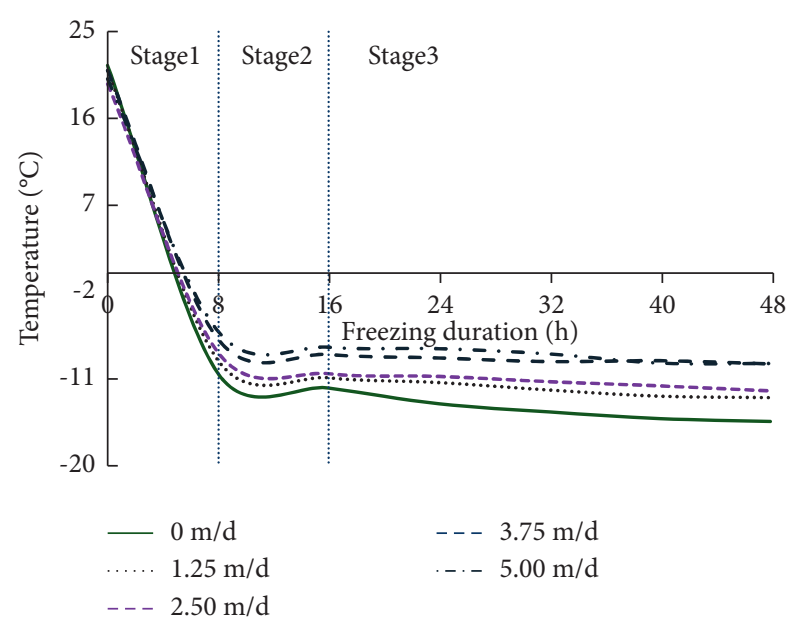

(b)

Figure 5: Time history of the brine temperature and freezing pipe wall temperature. (a) Brine in the freezing pipe in physical modelling. (b) Freezing pipe wall in physical modelling.

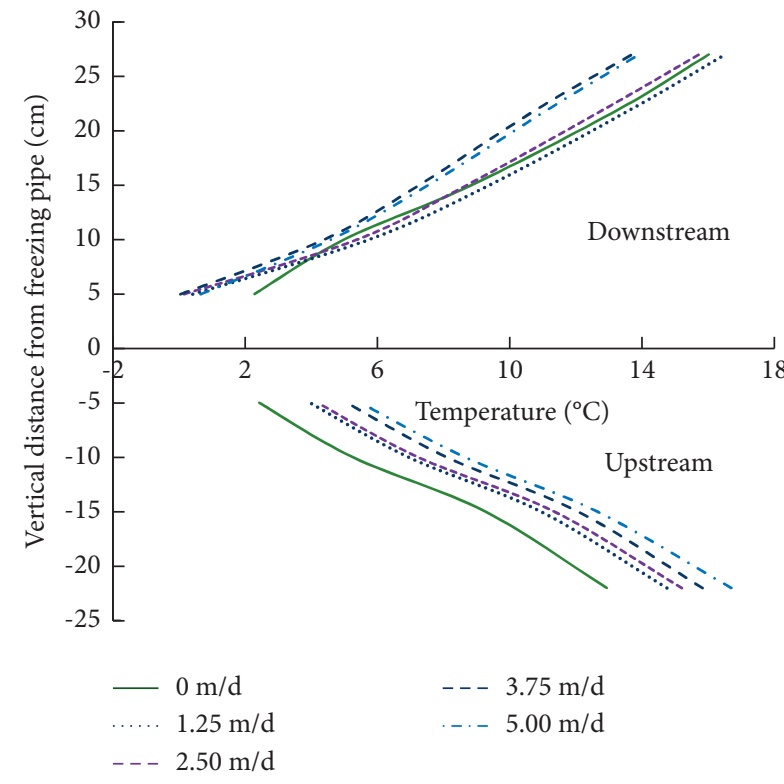

(a)

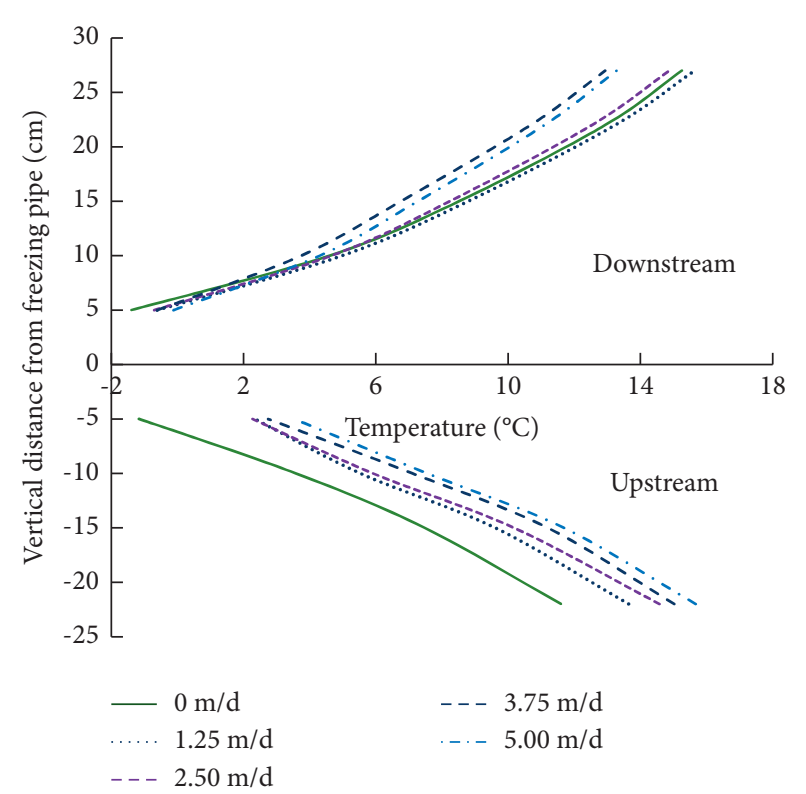

(b)

FIGURE 6: Upstream and downstream temperature distributions parallel to the seepage direction. (a) Temperature distribution of L3 survey line with a freezing duration of $8 \mathrm{~h}$. (b) Temperature distribution of L4 survey line with a freezing duration of $8 \mathrm{~h}$.

the frozen wall, as shown in Figure 8. As illustrated, the groundwater seepage flow rate significantly affects the time required for the frozen wall merging. The closure time of the frozen wall increases approximately linearly with an increase in the seepage flow rate. In particular, it consumes a lot of time when the groundwater seepage flow rate reaches $5.0 \mathrm{~m} / \mathrm{d}$.

3.4. Temperature Field of Gravel Formation. Four temperature survey lines were arranged to monitor the temperature evolution process of gravel formation, as shown in Figure 4. Based on a large number of measured temperatures, the temperature field of the gravel formation was obtained with the use of the linear interpolation method. Wang et al. [15] also adopted this method to obtain the temperature field.

Figures 9-11 show the temperature fields, which correspond to the freezing duration of $8 \mathrm{~h}$ and closure time of the frozen wall. The closure time of frozen wall is presented in Figure 8. Half of the gravel formation is attributed to the symmetry of the seepage flow and temperature. The isotherm line at $0^{\circ} \mathrm{C}$ is defined as the boundary of the frozen wall. As illustrated, the frozen wall develops in all directions with the freezing pipes as the centre, and they then merge after a certain freezing duration. The reason is that the heat provided by the freezing pipe is uniformly transmitted around the freezing pipe as the centre. 


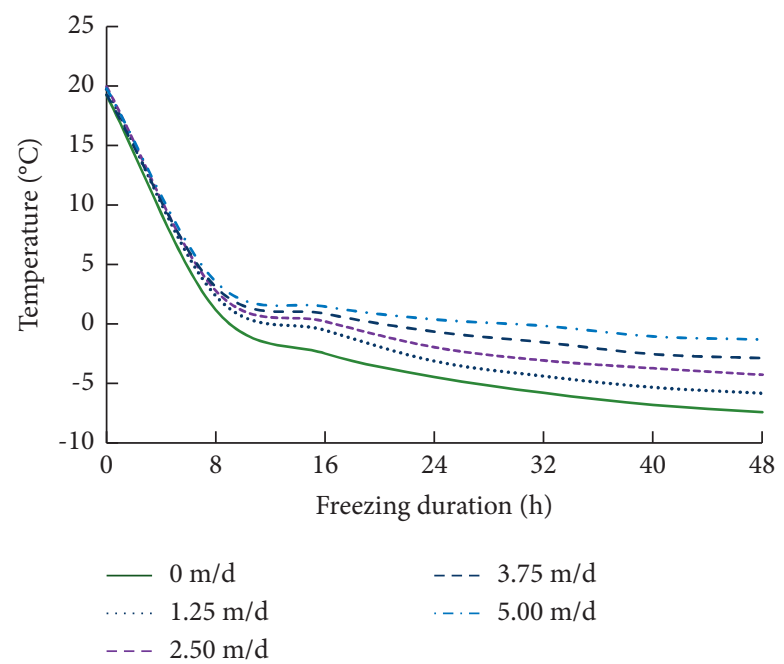

FIgURE 7: Time history of the temperature at survey points T1-6.

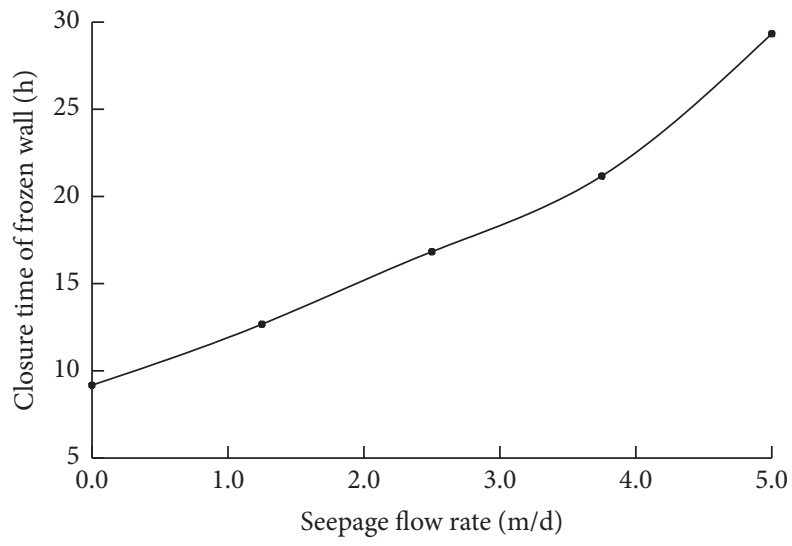

FIGURE 8: Closure time of frozen wall vs. groundwater seepage flow rate.

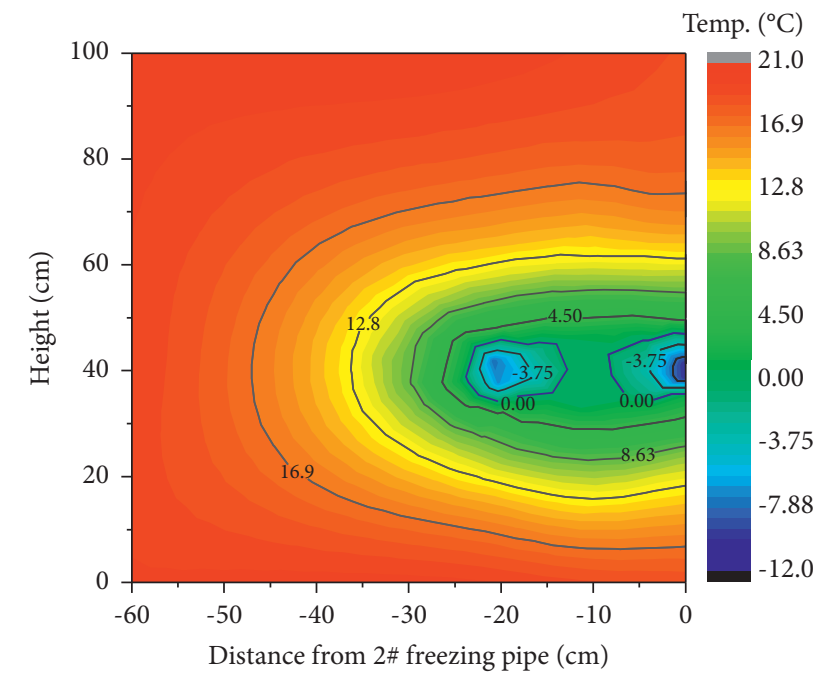

(a)

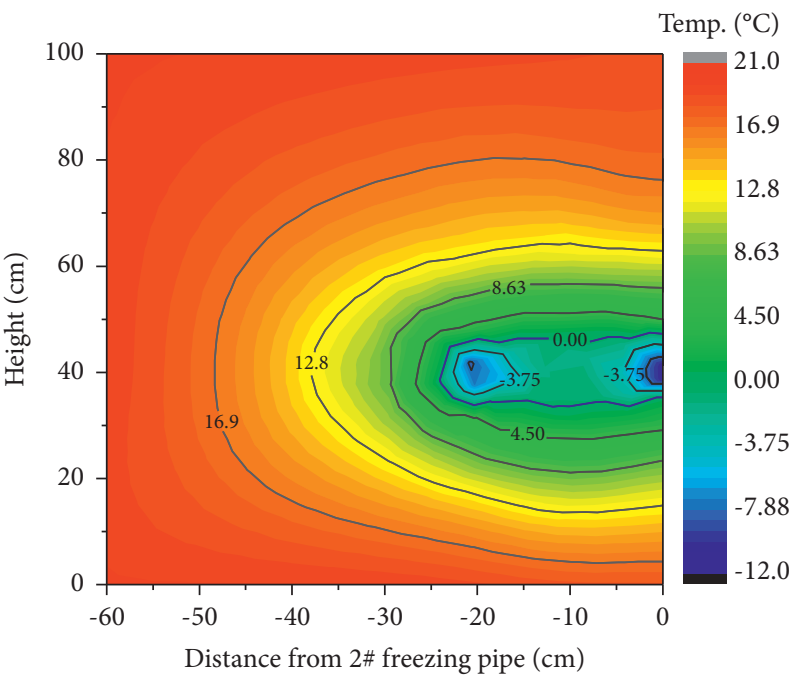

(b)

FIGURE 9: Temperature field of gravel formation under a nonseepage flow. (a) Freezing duration of $8 \mathrm{~h}$. (b) Closure time of frozen wall $9.8 \mathrm{~h}$. 


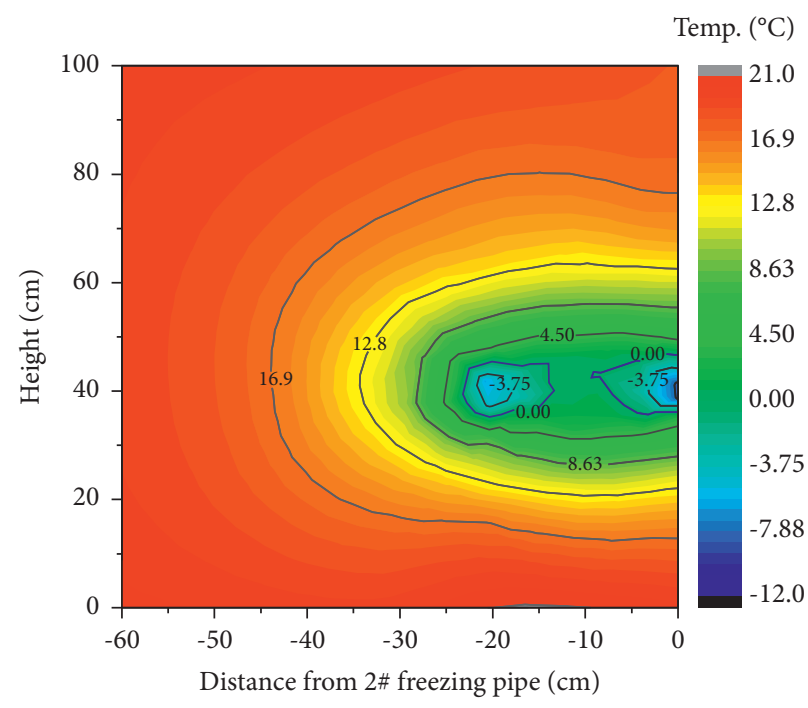

(a)

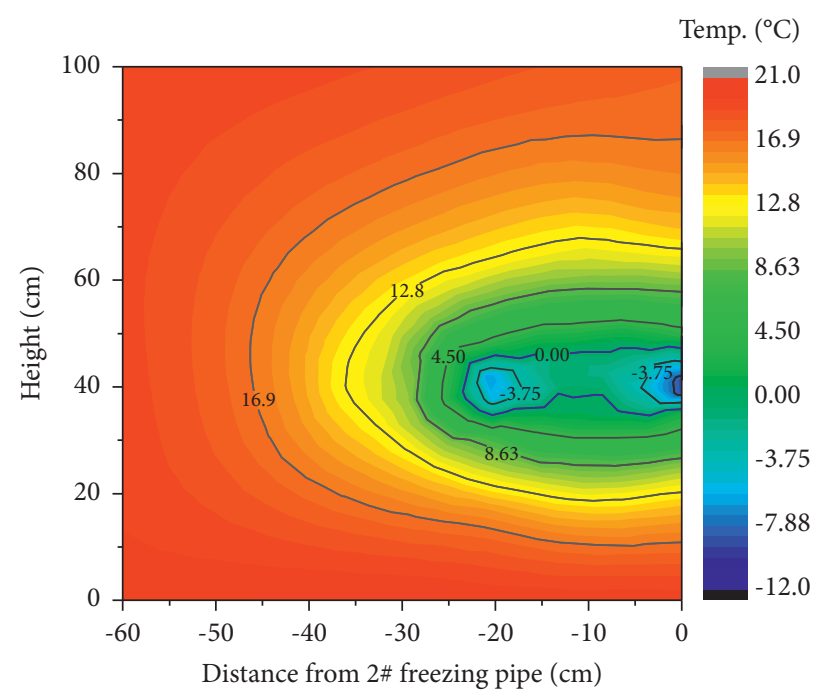

(b)

FIGURE 10: Temperature fields of gravel formation at a seepage flow rate of $2.5 \mathrm{~m} / \mathrm{d}$. (a) Freezing duration of $8 \mathrm{~h}$. (b) Closure time of frozen wall $16.8 \mathrm{~h}$.

Subject to the nonseepage flow condition, the frozen wall edges are neat and expand rapidly. When the frozen wall is merged, the frozen wall edges uniformly develop to the central position of the freezing pipes $1 \#$ and $2 \#$, and the merged frozen wall has a uniform thickness with no evident weak zones, as shown in Figure 9. The contours for the temperature field are evenly spaced, which indicates that the gravel formation temperature distribution presents a centrosymmetric characteristic, namely, upstream and downstream and left and right symmetries.

Because of the effect of groundwater seepage flow, the temperature distributions of the gravel formation in the upstream and downstream present asymmetric characteristics consistent with the conclusion drawn by Liu et al. (2017). In the upstream zone, the temperature under a flow rate of $2.5 \mathrm{~m} / \mathrm{d}$ is lower than that under a flow rate of $5.0 \mathrm{~m} / \mathrm{d}$ at the same height. On the contrary, the temperature under a flow rate of $2.5 \mathrm{~m} / \mathrm{d}$ is higher than that under a flow rate of $5.0 \mathrm{~m} / \mathrm{d}$ in the downstream zone. This is because the groundwater seepage flow carries more heat from the freezing pipes to the downstream zone. Moreover, the frozen wall edges present an irregular shape, as illustrated in Figures $10(\mathrm{a})$ and 11(a). In Figures $10(\mathrm{~b})$ and $11(\mathrm{~b})$, the merging position of the frozen wall shifts to the direction of the downstream zone. The frozen wall at the centre of the freezing pipes $1 \#$ and 2\# is the thinnest, and it presents an evident arched shape. Therefore, this position is the weakest zone in the entire frozen wall, whereas the deviation in the frozen wall towards the downstream zone increases.

3.5. Thickness of Merged Frozen Wall. As shown in Figure 12, the thicknesses of the frozen wall are obtained in accordance with the closure time. Setting the L1 survey line as the demarcation line, the total thickness is divided into the upstream and downstream thicknesses to better understand the influence of the groundwater seepage flow.

As shown, the upstream and downstream thicknesses are largely identical under the nonseepage flow. However, the groundwater seepage flow significantly influences the upstream and downstream thicknesses. The thickness difference between the downstream and upstream increases as a function of the seepage flow rate. Two types of effect are defined to explain the merging process of frozen wall: (1) the "scouring effect" refers to the process by which upstream groundwater carries away the heat of the freezing pipes to the downstream zone, and (2) the "water barrier effect" refers to the upstream frozen wall blocking the flowing of groundwater and results in the retention of heat.

The development of the upstream frozen wall is restricted because of the scouring effect of the groundwater seepage flow, particularly when the flow rate reaches $5.0 \mathrm{~m} / \mathrm{d}$. Hence, the upstream frozen wall thickness decreases linearly with the groundwater seepage flow rate, as shown in Figure 12. However, the downstream frozen wall thickness increases linearly with the groundwater seepage flow rate even beyond the nonseepage flow case. This is because the water barrier effect provided by the upstream frozen wall weakens the influence of the seepage flow on the development of the downstream frozen wall. Moreover, the water barrier effect leads to a decrease in the seepage flow rate around the downstream frozen wall, and the heat carried by the flowing water is more favourable to the development of the downstream frozen wall. The total thickness is the sum of the upstream and downstream frozen wall thicknesses. The total thickness decreases by more than $30 \%$ when the flow rate increases from 0 to $5.0 \mathrm{~m} / \mathrm{d}$. 


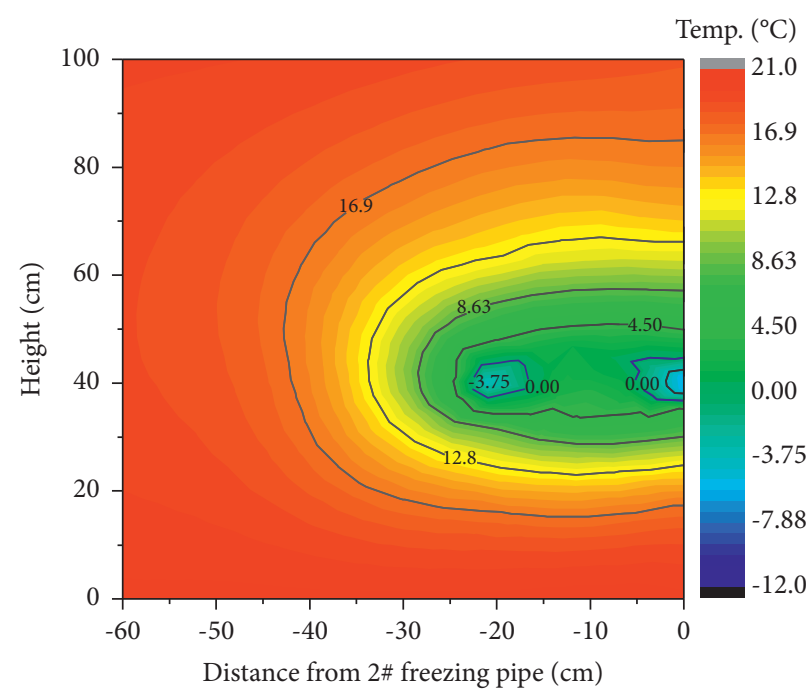

(a)

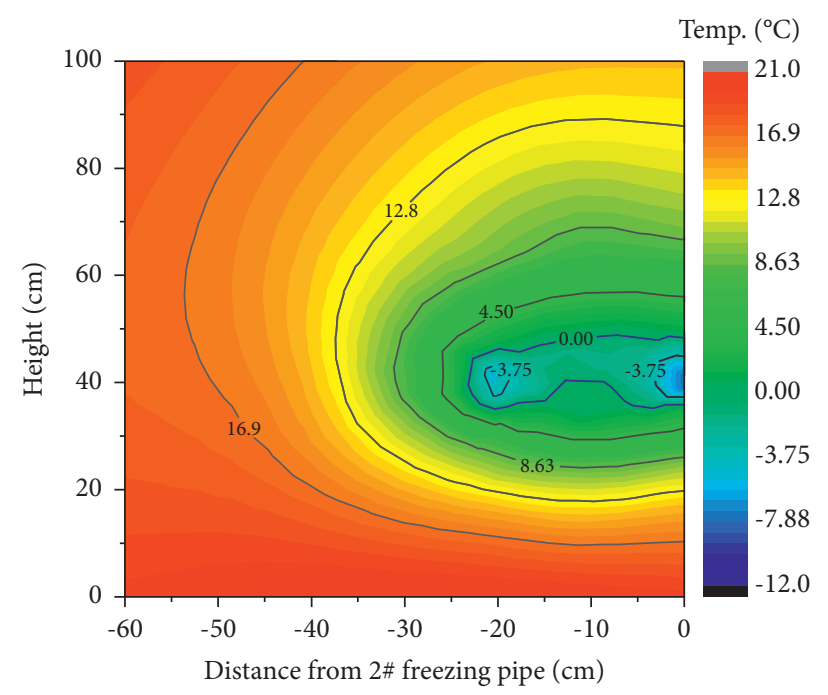

(b)

FIGURE 11: Temperature fields of gravel formation at a seepage flow rate of $5.0 \mathrm{~m} / \mathrm{d}$. (a) Freezing duration of $8 \mathrm{~h}$. (b) Closure time of frozen wall $29.3 \mathrm{~h}$.

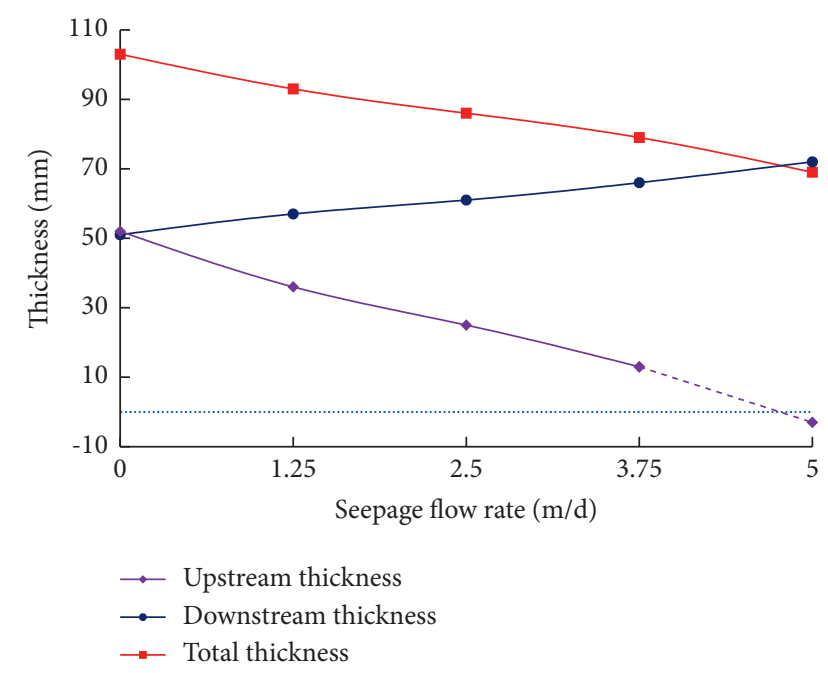

Figure 12: Frozen wall thickness corresponding to the closure time.

\section{Discussion}

The presented gravel soil consists of solid particles, liquid water, and ice lens [18]. In an AGF process, the effect of groundwater seepage flow on the frozen wall is important for application of AGF in such formations. For better comprehension of the mechanism, the entire AGF process undergone by the gravel formation is discussed.

(i) In water-rich gravel formation, the pore volume is assumed to be either fully saturated or partly occupied by ice lens and the rest by liquid water [13]. In comparison with fine grained soils, the liquid water is all free water with no bound water in the gravel soils $[15,19]$. The frozen wall is easier to merge without the groundwater seepage flow. (ii) The groundwater seepage flow results in heat exchange between the flowing water and freezing pipes, with some of the heat dissipated by the flowing water. Therefore, the scouring effect is dominant in the upstream zone, hinders the merging of the frozen wall, and then leads to a weak irregular zone and a longer closure time.

(iii) As the freezing duration increases, the pore volume is initially partly occupied by ice lens and the rest by the remaining water in the liquid form until it crystallizes into ice lens because of the increasing heat transmitted from the freezing pipes [13].

(iv) With the development of ice lens and upstream frozen wall, the water barrier effect becomes increasingly significant. The flowing water with a low rate is blocked around the downstream frozen wall and rapidly crystallizes into an ice lens until the frozen wall is merged. Therefore, the frozen wall presents an arched shape and shifts to the direction of the downstream zone at the end.

As the seepage flow rate increases, the frozen wall requires more time to freeze and heat (from the freezing pipes) to reach the designed thickness. However, these measures will lead to a significant increase in the project cost and do not address the weak irregular zone limitation of the frozen wall. Hence, we suggest that the layout parameters of the freezing pipes are optimised to reduce the interference of the groundwater seepage flow for a safe and economical design. As calculated by Marwan et al. [13], an optimised arrangement of the freezing pipes can help reduce the freezing time under different seepage flow rates in fine sand formations. Hence, additional studies should be conducted to integrate a more useful optimisation algorithm $[20,21]$ to verify its adaptability in the case of gravel formations, to extend the application scope of the AGF method in water-rich formations. 


\section{Conclusions and Outlook}

The AGF method is a process that involves the coupling action of groundwater and temperature. In particular, the groundwater seepage flow cannot be ignored in water-rich gravel formations. To understand the AGF process in depth, we thoroughly investigated the coupling effect of groundwater seepage flow and temperature in the gravel formation. The following are the conclusions drawn from the results:

(1) The groundwater seepage flow intensifies the heat exchange between the flowing water and freezing pipes, thus reducing the work efficiency of the freezing pipes and the closure time required for the frozen wall.

(2) The upstream and downstream temperature distributions exhibit symmetric characteristics in the case of nonseepage flow. However, the isotherm lines in the upstream zone appear more intense, and those in the downstream zone appear sparser subject to the action of the groundwater seepage flow.

(3) The closure time of the frozen wall increases as a function of the groundwater seepage flow rate. The flowing water causes an uneven development of the frozen wall, which presents an arched shape. This type of uneven characteristic is significant at highgroundwater seepage flow rates.

(4) The groundwater seepage flow exhibits a scouring effect on the upstream frozen wall, whereas the water barrier effect provided by the upstream frozen wall is conducive to the development of the downstream frozen wall. The total thickness of the frozen wall decreases by more than $30 \%$ when the flow rate increases from 0 to $5.0 \mathrm{~m} / \mathrm{d}$.

The following aspects can be investigated in future studies:

(1) The effects of the spacing of the freezing pipes on the coupling of the groundwater seepage and heat flow

(2) Algorithms for optimising the arrangement of the freezing pipes in the presence of groundwater seepage flow

\section{Data Availability}

All data included in this study are available upon request by contact with the corresponding author.

\section{Conflicts of Interest}

The authors declare that they have no conflicts of interest.

\section{Acknowledgments}

This research was funded by the National Natural Science Foundation of China (NSFC) (grant nos. 51978426 and 51708369) and Natural Science Foundation of Hebei Province (under grant nos. E2021210007 and E2021210128). The authors would like to thank the language editor for English language editing.

\section{References}

[1] H. L. Jessberger, "Theory and application of ground freezing in civil engineering," Cold Regions Science and Technology, vol. 3, no. 1, pp. 3-27, 1980.

[2] M. Vitel, A. Rouabhi, M. Tijani, and F. Guérin, "Modeling heat and mass transfer during ground freezing subjected to high seepage velocities," Computers and Geotechnics, vol. 73, pp. 1-15, 2016.

[3] G. Russo, A. Corbo, F. Cavuoto, and S. Autuori, "Artificial Ground Freezing to excavate a tunnel in sandy soil. Measurements and back analysis," Tunnelling and Underground Space Technology, vol. 50, pp. 226-238, 2015.

[4] W. Fan and P. Yang, "Ground temperature characteristics during artificial freezing around a subway cross passage," Transportation Geotechnics, vol. 20, Article ID 100250, 2019.

[5] J. Zhou and Y. Tang, "Centrifuge experimental study of thaw settlement characteristics of mucky clay after artificial ground freezing,” Engineering Geology, vol. 190, pp. 98-108, 2015.

[6] A. Rouabhi, E. Jahangir, and H. Tounsi, "Modeling heat and mass transfer during ground freezing taking into account the salinity of the saturating fluid," International Journal of Heat and Mass Transfer, vol. 120, pp. 523-533, 2018.

[7] G. Hirose and Y. Ito, "Experimental estimation of permeability of freeze-thawed soils in artificial ground freezing," Procedia Engineering, vol. 189, pp. 332-337, 2017.

[8] E. Pimentel, A. Sres, and G. Anagnostou, "Large-scale laboratory tests on artificial ground freezing under seepage-flow conditions," Géotechnique, vol. 62, no. 3, pp. 227-241, 2012.

[9] P. E. Frivik and G. Comini, "Seepage and heat flow in soil freezing," Journal of Heat Transfer, vol. 104, no. 2, pp. 323-328, 1982.

[10] R. A. Sudisman, M. Osada, and T. Yamabe, "Heat transfer visualization of the application of a cooling pipe in sand with flowing pore water," Journal of Cold Regions Engineering, vol. 31, no. 1, pp. 1-27, 2017.

[11] K. S. Prabhata and K. Deepak, "Design of minimum seepage loss canal sections with drainage layer at shallow depth," Journal of Irrigation and Drainage Engineering, vol. 130, no. 2, pp. 166-170, 2004.

[12] M. Vitel, A. Rouabhi, M. Tijani, and F. Guérin, “Thermohydraulic modeling of artificial ground freezing: application to an underground mine in fractured sandstone," Computers and Geotechnics, vol. 75, pp. 80-92, 2016.

[13] A. Marwan, M.-M. Zhou, M. Zaki Abdelrehim, and G. Meschke, "Optimization of artificial ground freezing in tunneling in the presence of seepage flow," Computers and Geotechnics, vol. 75, pp. 112-125, 2016.

[14] T.-L. Wang, H.-H. Wang, H.-F. Song, Z.-R. Yue, and Z.-H. Guo, "Effects of cement content and grain-size composition on engineering properties of high-speed-railway macadam subgrade," Cold Regions Science and Technology, vol. 145, pp. 21-31, 2018.

[15] T.-L. Wang, Y.-Z. Zhang, M. Wei, B.-Y. Cheng, and A. Wen, "Investigation of liquid and vapor migration in coarse-grained soil during open-system step-freezing test," Cold Regions Science and Technology, vol. 165, Article ID 102816, 2019.

[16] Ministry of Construction of the People's Republic of China, Standard for Engineering Classification of Soil, China Plans Publishing House, Beijing, China, 2008.

[17] T.-L. Wang, Z.-R. Yue, C. Ma, and Z. Wu, "An experimental study on the frost heave properties of coarse grained soils," Transportation Geotechnics, vol. 1, no. 3, pp. 137-144, 2014. 
[18] M. M. Zhou and G. Meschke, "A three-phase thermo-hydromechanical finite element model for freezing soils," International Journal for Numerical and Analytical Methods in Geomechanics, vol. 37, no. 18, pp. 3173-3193, 2013.

[19] J. Zhou and Y. Tang, "Artificial ground freezing of fully saturated mucky clay: thawing problem by centrifuge modeling," Cold Regions Science and Technology, vol. 117, pp. 1-11, 2015.

[20] M. Dorigo and C. Blum, "Ant colony optimization theory: a survey," Theoretical Computer Science, vol. 344, no. 2-3, pp. 243-278, 2005.

[21] M. López-Ibáñez and T. Stützle, "An experimental analysis of design choices of multi-objective ant colony optimization algorithms," Swarm Intelligence, vol. 6, no. 3, pp. 207-232, 2012. 